\title{
Spectral bandwidth and phase effects of resonantly excited ultrafast surface plasmon pulses
}

\author{
S. E. Yalcin, Y. Wang, and M. Achermann* \\ Physics Department, University of Massachusetts, Amherst, Massachusetts 01003, USA.
}

We report on the detailed analysis of femtosecond surface plasmon polariton (SPP) pulse generation under resonant excitation. Using prism coupling technique we excite femtosecond SPP pulses at a gold/air interface with ultrafast laser pulses. We show that the photon-SPP coupling is a resonant process with a finite spectral bandwidth that causes a spectral phase shift and a narrowing of the SPP pulse spectrum. Both effects result in a temporal pulse broadening and, therefore, set a lower limit on the duration of ultrafast SPP pulses with consequences for ultrafast SPP applications.

PACS numbers: 73.20.Mf; 41.20.Jb; 42.65.Re

*e-mail: achermann@physics.umass.edu 
Surface-bound electromagnetic waves that propagate at metal-dielectric interfaces have attracted much attention due to their sensitivity to interface changes and their ability to guide and confine light beyond the diffraction limit. ${ }^{1}$ These surface plasmon polaritons (SPPs) have been utilized in SPP resonance sensors and imaging techniques ${ }^{2}$ and, more recently, to monitor and control ultrafast charge carrier and energy dynamics in thin films. ${ }^{3}$ Moreover, SPP building blocks are thought to be attractive components for photonic circuits, ${ }^{4}$ because they allow for high packing densities $^{5}$ and strong non-linearities. ${ }^{6}$ For both time-resolved SPP sensors and high-speed SPP circuits it is key to understand excitation ${ }^{7}$ and propagation ${ }^{8-10}$ of ultrafast SPPs.

The SPP is an electromagnetic wave that is bound to a metal/dielectric interface by coupling to the free electrons of the metal. As a consequence, the SPP wave vector, $k_{S P P}$, is larger than the wave vector in the dielectric medium. Whereas the large $k_{S P P}$ and the confinement to an interface are advantageous for plasmonic circuits, ${ }^{4}$ the downside is that SPP excitation directly from the dielectric side of the interface is prohibited through momentum conservation. However, the momentum mismatch can be compensated for with either a grating or a small scattering object on the metal surface. Alternatively, SPP excitation is achieved by sending p-polarized light through a high refractive index prism on the back side of a thin metal film. In this widely used prism coupling technique [inset of Fig. 1(a)] introduced by Kretschmann ${ }^{11}$ the SPP is excited on the front side of the metal film which faces a lower refractive index environment. The SPP excitation is a resonant process and occurs when the in-plane wave vector of the incident light within the prism matches $k_{S P P}$. Here, we show that the resonance condition affects ultrafast SPP pulse generation by modifying the spectral amplitude and phase of the SPP pulse. The two effects cause a temporal broadening of the ultrafast SPP pulses and set a lower limit to the SPP 
pulse width with consequences for ultrafast SPP spectroscopy techniques, ${ }^{3}$ high-speed plasmonic devices, ${ }^{4}$ non-linear SPP concepts, ${ }^{6}$ and ultrafast nanoplasmonics. ${ }^{12}$

In the prism configuration the resonance condition for the coupling between photons and SPP at a metal/dielectric interface with dielectric functions $\varepsilon_{m}$ and $\varepsilon_{d}$, respectively, is:

$$
n_{p r i s m} \sin \left(\theta_{S P P}\right)=\sqrt{\frac{\varepsilon_{d} \varepsilon_{m}}{\varepsilon_{d}+\varepsilon_{m}}},
$$

in which $n_{\text {prism }}$ is the refractive index of the prism and $\theta_{S P P}$ is the coupling angle [see inset of Fig. 1(a)]. Using an appropriate metal film thickness (typically $\sim 50 \mathrm{~nm}$ for gold and silver films in the visible and near-infrared wavelength range), the energy of the incident light can be converted very efficiently into SPPs. Such energy conversion appears as a reflectance close to zero at the resonance angle, $\theta_{S P P}[$ Fig. 1(a)]. Since the coupling condition of equation 1 and the angle dependence of the reflectance are very sensitive to $\varepsilon_{d}$, this SPP prism configuration has been used for sensing ${ }^{2}$ and, more recently, for studying ultrafast dynamics in thin films. ${ }^{3}$

The described resonance condition for SPP excitation is not only apparent in momentum space but also in frequency space as a result of the frequency dependent dielectric functions. In Fig. 1(b) and 1(c) we show the calculated (see Ref. 13 for material parameters) and measured (using a tungsten halogen lamp) reflectance of a $50 \mathrm{~nm}$ thick gold film as a function of incidence angle and photon energy. At a given excitation angle the reflectance drops within a finite photon energy range that defines the SPP coupling bandwidth. While for continuous wave (cw) applications this consideration has little relevance, it is expected that the broad spectrum of ultrafast pulses will be strongly affected by the finite spectral bandwidth of the resonant excitation process. 
We study the excitation process of ultrafast SPP pulses using sub 25 fs laser pulses from a Ti:sapphire oscillator. The p-polarized laser pulses are close to bandwidth limited with a spectral full width at half maximum (FWHM) of $56 \mathrm{~nm}$, centered about $820 \mathrm{~nm}$. Using the prism setup we excite ultrafast SPP pulses at the metal/air interface of a $50 \mathrm{~nm}$ thick gold film. The resonant SPP excitation condition is found by tuning the incidence angle (corresponding to tuning the inplane wave vector) of the ultrafast laser beam and measuring its reflected power. The reflectivity drops at the coupling angle [Fig. 1(a)]; however, compared to calculations ${ }^{14}$ the measured reflectance dip is less pronounced and suggests incomplete coupling between the femtosecond laser pulse and the SPP. Interestingly, the spectrum of the reflected pulse at the optimal coupling angle is not just uniformly suppressed but strongly modulated (Fig. 2). At a fixed incidence angle only a finite spectral bandwidth of the femtosecond laser pulse couples to the SPP. For example, the reflected spectrum of the excitation pulse at the optimal coupling angle, $\theta_{S P P}$, shows a double peak [Fig. 2(a)], which is the result of the spectral bandwidth of the photon-SPP coupling that is narrower than the spectral width of the ultrafast laser pulse. In addition, the spectrum of the reflected pulse depends strongly on the incidence angle. The missing part of the reflected spectrum that indicates SPP coupling moves to higher photon energies with increasing incidence angle / higher in-plane wave vector. Such behavior is consistent with the dispersion relation of SPPs.

The limited spectral bandwidth of the prism-coupling technique also becomes evident from spectral measurements of scattered SPPs. Since the Au film has a finite surface roughness, the SPP scatter into free space propagating light that we collected and analyzed. Assuming that the SPP scattering process varies only slightly over the wavelength range of interest, the scattered spectra represent the ultrafast SPP pulse spectra. Fig. 2(b) shows the spectrum of scattered SPP 
pulses that we determine to be $22 \%$ narrower than the incident excitation pulse spectrum. This is a direct consequence of the spectral filtering effect of the resonant coupling process. The scattered SPP spectrum matches closely to the difference spectrum of the incident and the reflected pulse spectra [Fig. 2(b)], thus confirming the assumption that the reflectance dip is due to SPP coupling. It is noteworthy that the correspondence between reflected and scattered SPP spectra illustrates nicely that the characteristics of SPP and reflected excitation pulses are complementary and, therefore, reflected excitation pulses are well suited to analyze SPP pulses.

Ultimately, the spectral width of the SPP pulse is limited by the spectral bandwidth of the SPP prism coupling that we calculated as $\sim 120 \mathrm{meV}$ and $\sim 40 \mathrm{meV}$ for gold and silver thin films, ${ }^{15}$ respectively, in the near infrared wavelength range [Fig. 2(c)]. The narrower bandwidth of silver films compared to gold films is attributed to lower losses (smaller imaginary part of the dielectric function) and is consistent with a higher quality factor of the resonant excitation process in $\mathrm{Ag}$ films. Since the finite spectral bandwidth of the SPP coupling process limits the spectral width of the SPP pulses, it also sets a lower limit for the SPP pulse duration. The pulse duration limit is determined by the time-bandwidth product and is independent of the pulse duration of the excitation pulse. In case the entire spectral bandwidth is used for SPP excitation and a spectrally flat phase is considered, one would obtain a lower limit for SPP pulse durations of $\sim 11$ and $\sim 33$ fs for gold and silver films, respectively. However, since ultrafast SPP pulse generation is a resonant process, we expect a spectral phase shift around the resonance frequency.

To test this hypothesis we employ spectral interferometry to measure the phase of the reflected pulse that provides a good fingerprint of the SPP pulse (scattered SPP light can not be used for phase measurements, since phase information is lost in the random scattering process). We let the reflected pulse interfere with a reference pulse that is split off of the excitation laser 
beam before the prism setup. From the interferogram [Fig. 3(a)] and by using Fourier analysis we extract the phase difference between the reflected and reference pulse. ${ }^{16}$ The measured phase is the sum of the phase change induced by the SPP excitation and the phase contribution from the transmission of the pulse through the prism. To determine the latter phase we measure a second interferogram with the same prism setup but without a metal film (total internal reflection (TIR) configuration). The phase associated with the SPP pulse excitation [see Fig. 3(a)] is then essentially the difference between the TIR phase and the SPP excitation phase. ${ }^{17}$ Around the SPP excitation photon energy we find a significant phase shift of $\sim \pi / 2$ within $50 \mathrm{meV}$. The spectral phase shift is characteristic of a resonant process and matches very well the calculated phase of the reflected electric field. The SPP pulse itself is experiencing a phase shift as shown in Fig. 3(a) that will further extend the SPP pulse duration in addition to the spectral narrowing effect. Both spectral narrowing and phase shift effects are direct consequences of the resonant excitation process that we used to generate ultrafast SPP pulses.

The spectral amplitude and phase modulations limit the duration of ultrafast SPP pulses and, in addition, affect the time resolution of pump-probe experiments that utilize SPP excitation. To study the latter effect we determine the duration of the reflected pulse using the interferometric autocorrelation (IAC) technique. In Fig. 3(b) and 3(c) we display the IAC of the reflected pulse under resonant SPP excitation and the IAC of a reference pulse under TIR condition. The IAC of the reference pulse is characteristic of a nearly bandwidth-limited pulse of sub 25 fs pulse duration. In contrast, the IAC of the reflected pulse under resonant SPP excitation shows a short central pulse and significant satellite pulses. The central pulse is slightly shorter than the reference pulse as a result of the larger pulse spectrum; however, the short pulse duration comes at the expense of significant satellite pulses that are a consequence of the above measured 
spectral amplitude and phase modulation. Such satellite pulses that extend beyond 50 fs have to be taken into account when analyzing ultrafast pump-probe measurements that employ the prism configuration.

In conclusion, we have shown that the excitation of ultrafast SPP is strongly affected by the conventionally used prism coupling technique. This resonant excitation process causes spectral narrowing and phase shifts and leads to temporally broadened SPP pulses. Our discussion here is not restricted to the prism coupling technique, but is common to any SPP excitation process that uses resonant coupling between photons and surface plasmons. It has to be noted that both spectral narrowing and spectral phase shift can, in principle, be compensated. The former effect can be avoided by using an excitation pulse with appropriate angle dispersion, and the latter effect by modulating the phase of the incident field using pulse shaping techniques. Such techniques have previously been suggested to manipulate plasmon fields in metal nanostructures. $^{18}$

The authors thank Dr. L. Gallmann for valuable discussions on phase measurements. This work was supported by the NSF Center for Hierarchical Manufacturing (NSEC DMI-0531171). 


\section{References}

1. W.L. Barnes, A. Dereux, and T.W. Ebbesen, Surface plasmon subwavelength optics, Nature 424, 824 (2003).

2. J. Homola, S.S. Yee, and G. Gauglitz, Surface plasmon resonance sensors: review, Sensors Actuat. B 54, 3 (1999).

3. G.P. Wiederrecht, J.E. Hall, and A. Bouhelier, Control of Molecular Energy Redistribution Pathways via Surface Plasmon Gating, Phys. Rev. Lett. 98, 83001 (2007).

4. R. Zia, J.A. Schuller, A. Chandran, and M.L. Brongersma, Plasmonics: the next chip-scale technology, Materials Today 9, 20 (2006).

5. H.T. Miyazaki, and Y. Kurokawa, Squeezing Visible Light Waves into a 3-nm-Thick and 55-nm-Long Plasmon Cavity, Phys. Rev. Lett. 96, 97401 (2006).

6. D. Pacifici, H.J. Lezec, and H.A. Atwater, All-optical modulation by plasmonic excitation of CdSe quantum dots, Nature Photon. 1, 402-406 (2007).

7. Y. H. Liau, S. Egusa, and N. F. Scherer, Ultrafast interferometric measurements of plasmonic transport in photonic crystals, Optics Letters 27, 857-859 (2002).

8. D. S. Kim, S. C. Hohng, V. Malyarchuk, Y. C. Yoon, Y. H. Ahn, K. J. Yee, J.W. Park, J. Kim, Q. H. Park, and C. Lienau, Microscopic Origin of Surface-Plasmon Radiation in Plasmonic Band-Gap Nanostructures, Phys. Rev. Lett. 91, 143901 (2003).

9. R. Rokitski, K.A. Tetz, and Y. Fainman, Propagation of Femtosecond Surface Plasmon Polariton Pulses on the Surface of a Nanostructured Metallic Film: Space-Time Complex Amplitude Characterization, Phys. Rev. Lett. 95, 177401 (2005).

10. A. Kubo, N. Pontius, and H. Petek, Femtosecond Microscopy of Surface Plasmon Polariton Wave Packet Evolution at the Silver/Vacuum Interface, Nano Lett. 7, 470-475 (2007).

11. E. Kretschmann, Die Bestimmung optischer Konstanten von Metallen durch Anregung von Oberflachenplasmaschwingungen, Z. Phys. 241, 313-324 (1971).

12. M. I. Stockman, Ultrafast nanoplasmonics under coherent control, New J. Phys. 10, 025031 (2008).

13. P.B. Johnson, and R.W. Christy, Optical Constants of the Noble Metals, Phys. Rev. B 6, 4370-4379 (1972). 
14. The reflectivity was calculated for p-polarized light at the center photon energy of the excitation pulse.

15. For the calculations we considered $n_{\text {prism }}=1.52$ independent of the wavelength, since the spectral bandwidth is rather insensitive to $n_{\text {prism }}$.

16. M. Takeda, H. Ina, and S. Kobayashi, Fourier-transform method of fringe-pattern analysis for computer-based topography and interferometry, J. Opt. Soc. Am. 72, 156-160 (1982).

17. The phase of the reflection in the TIR configuration is wavelength independent at $\theta_{S P P}$, since $\theta_{S P P}$ is significantly larger than the critical angle for TIR. A small difference in optical path length between the SPP and TIR configurations led to a linear spectral phase difference that we subtracted.

18. M.I. Stockman, S.V. Faleev, and D.J. Bergman, Coherent Control of Femtosecond Energy Localization in Nanosystems, Phys. Rev. Lett. 88, 67402 (2002). 


\section{Figure Legends}

Fig. 1. (a) Calculated ${ }^{14}$ (solid line) and measured (filled circles) reflectivity of a femtosecond laser pulse off a gold film in the vicinity of the SPP coupling angle, $\theta_{S P P}$. Inset: Schematic of the prism configuration. Calculated (b) and measured (c) reflectivity as a function of photon energy and incidence angle.

Fig. 2. (color online) (a) Spectra of reflected femtosecond laser pulses at different incidence angles. (b) Comparison of scattered SPP pulse spectrum (black line), incident pulse spectrum (dashed line), reflected pulse spectrum at optimal SPP coupling angle (green line), and difference spectrum (square markers) between incident and reflected pulse spectra. (c) Spectral bandwidth (FWHM) of the SPP excitation by prism coupling for gold films (orange line) and silver films (black line).

Fig. 3. (color online) (a) The measured phase (red solid line) of the reflected pulse at resonant SPP excitation is extracted by Fourier analysis from the spectral interferogram (black line). As a comparison we show the calculated phase of the reflected field (red dashed line) and the SPP field (blue dashed line). A spectrally flat offset was added to the measured and calculated phases to overlap them. Interferometric autocorrelation (IAC) of the reflected pulse (b) under SPP excitation condition, indicating satellite pulses that are absent in (c), the reference IAC, taken in the TIR configuration. 

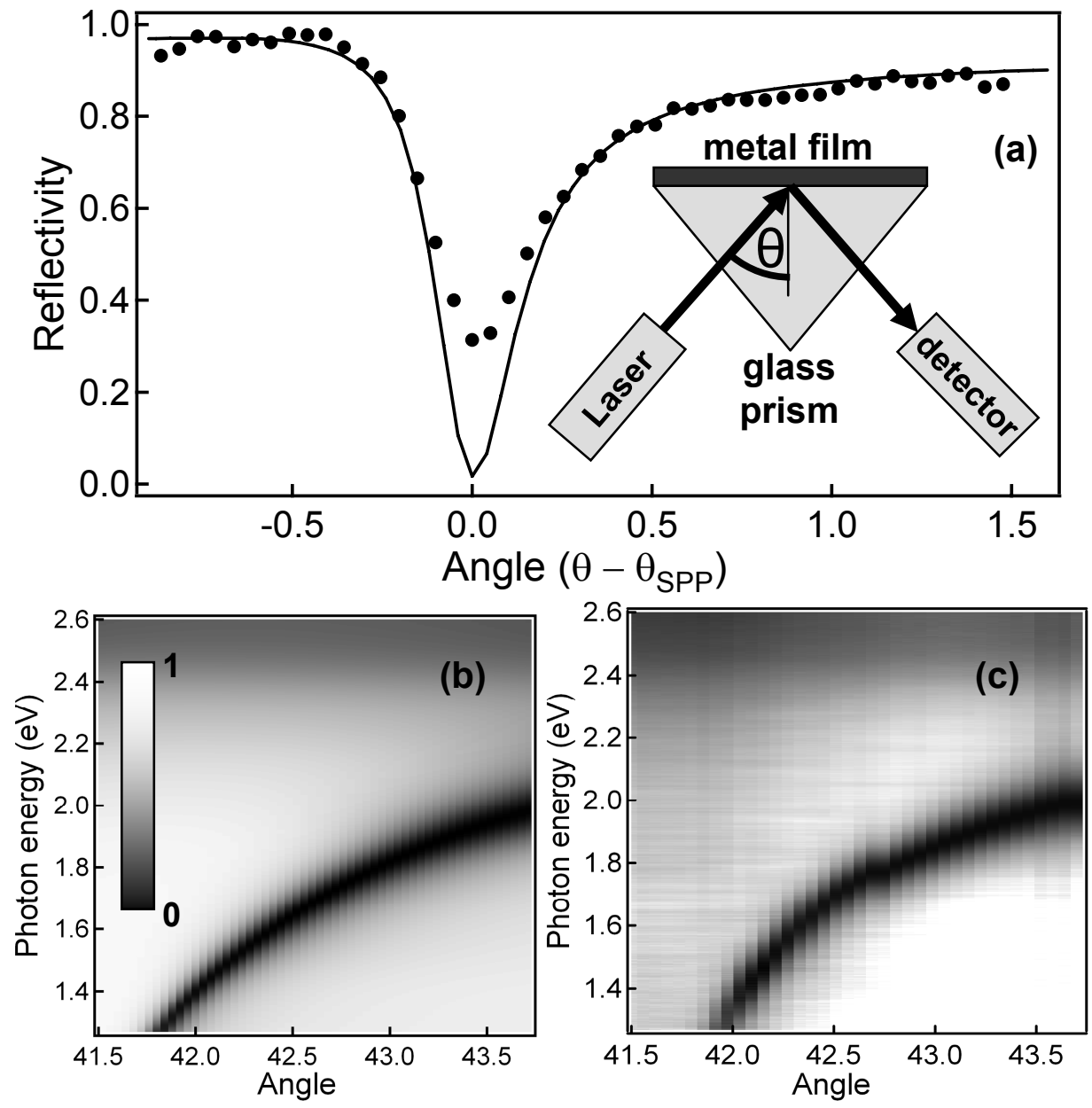

Figure 1

S. E. Yalcin et al.,

Spectral bandwidth and phase effects of resonantly excited ultrafast surface plasmon pulses. 

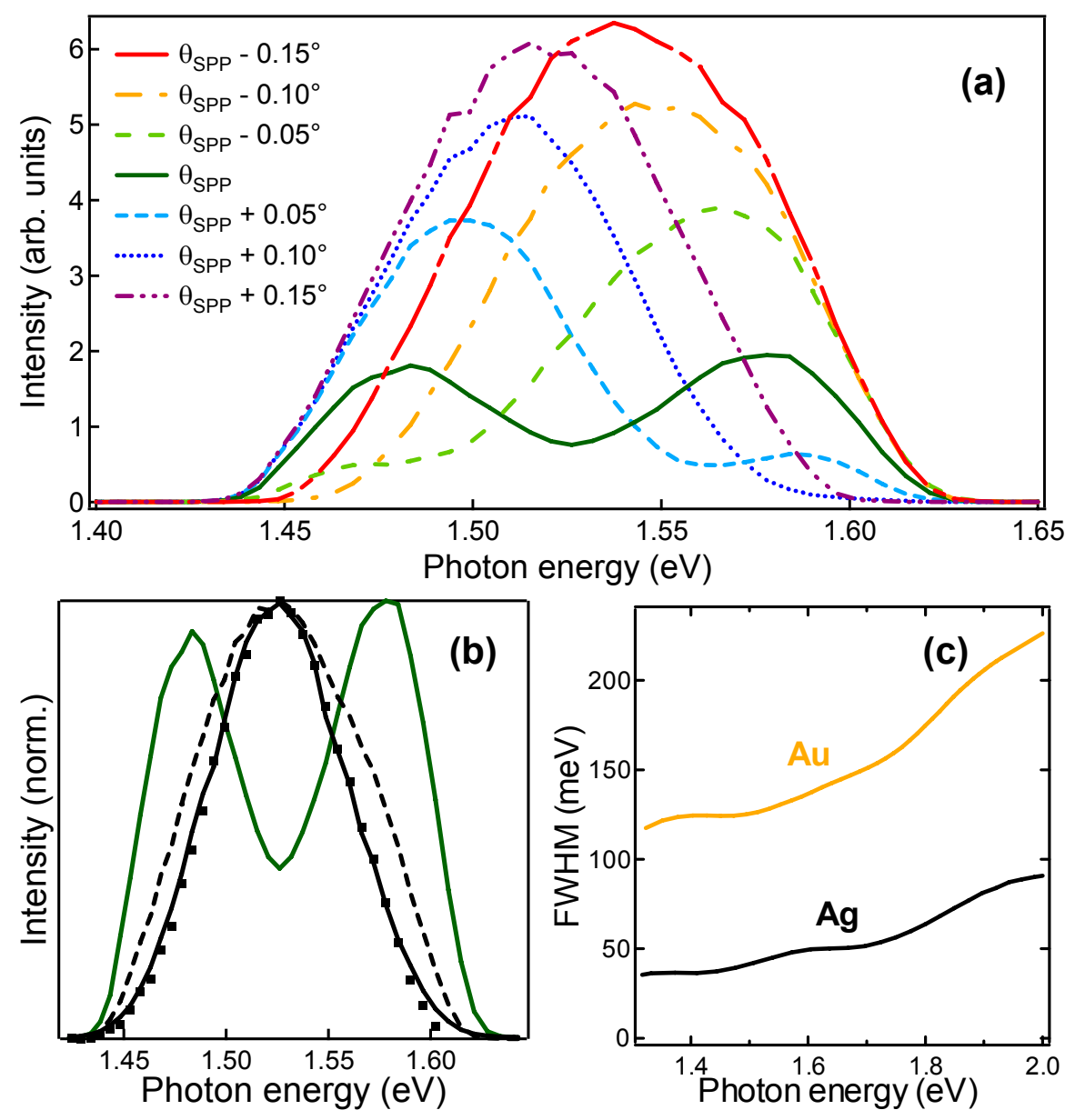

Figure 2

S. E. Yalcin et al.,

Spectral bandwidth and phase effects of resonantly excited ultrafast surface plasmon pulses. 

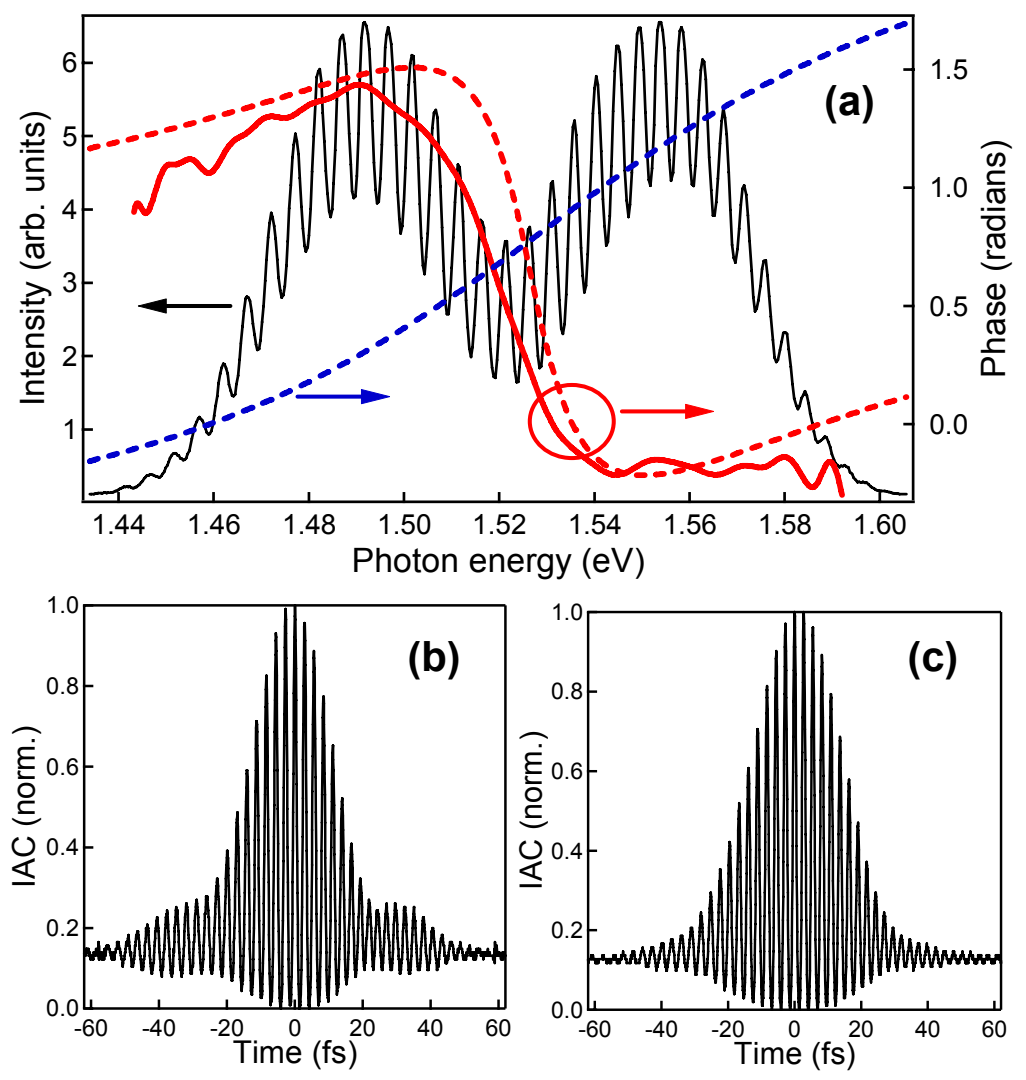

Figure 3

S. E. Yalcin et al.,

Spectral bandwidth and phase effects of resonantly excited ultrafast surface plasmon pulses. 